\title{
Dialisi peritoneale: dialisi marginale? Opinioni a confronto
}

\author{
Renzo Scanziani, Sara Auricchio, Marco Pozzi, Beatrice Dozio \\ Divisione Nefrologia e Dialisi, Azienda Ospedaliera di Desio e Vimercate, Presidio di Desio, Desio (MB)
}

\begin{abstract}
Peritoneal dialysis: marginal dialysis? A comparison of opinions
Abstract. Peritoneal dialysis (PD) offers many advantages over hospital-based hemodialysis, including a better quality of life. Despite the scientific and technological developments, PD is generally underused, a fact that can be attributed not only to a lack of knowledge and education amongst renal clinicians and nurses, but also to an inappropriate PD outpatient management by dedicated staff. To expand the diffusion of PD we need more scientific research, training, and implementation of human resources.
\end{abstract}

Key words: Integrated assistance, Training, Region

Conflict of interest: None.

Financial support: None.

Accettato: 17 Dicembre 2013

Dalla sua introduzione, ormai parecchi anni fa, la dialisi peritoneale ha compiuto molti progressi e va considerata a tutti gli effetti un'opzione più che valida per il trattamento sostitutivo dell'uremia, con un'ottima sopravvivenza, addirittura maggiore dell'emodialisi nei primi due anni di trattamento, e una qualità di vita da parte del paziente, per certi versi, migliore. Negli ultimi decenni, operatori sanitari (medici e infermieri), aziende farmaceutiche e istituzioni hanno compiuto sforzi significativi per incrementare la penetranza della dialisi peritoneale. Gli operatori sanitari hanno sostenuto la ricerca di base sul trattamento e sulle complicanze. Le ditte hanno contribuito, sviluppando soluzioni biocompatibili e cycler tecnologicamente avanzati (apparecchiature e sistemi di connessione sempre più sicuri, maneggevoli e di facile comprensione) e promuovendo un'assistenza integrata al paziente domiciliare (training, assistenza diretta per le problematiche tecniche e consegna del materiale). Alcune regioni hanno sponsorizzato la metodica con incentivi economici a sostegno del paziente avviato al trattamento dialitico.

Tuttavia, la diffusione della dialisi peritoneale resta limitata. In Italia, la percentuale dei pazienti prevalenti non è aumentata e, in alcune realtà, si è assistito a una riduzione dei pazienti sia incidenti che prevalenti.

Quali sono i fattori che rendono la dialisi peritoneale così marginale?

In primis, i nefrologi. Il medico deve essere guida, per i pazienti, nella scelta e nel mantenimento in vita della metodica dialitica. E, per svolgere tale ruolo, deve ispirare fiducia e mostrare competenza.

La mancanza di convinzione e la scarsa decisione nel proporre il trattamento peritoneale di molti colleghi scettici, che "non credono" nella validità della metodica, sono i principali ostacoli all'incremento della penetranza della metodica sul nostro territorio.

Lo scarso aggiornamento di tanti colleghi può rendere difficile l'approccio al malato non solo nella gestione delle complicanze, ma anche nei casi più semplici, nella routine.

L'assenza di un'adeguata formazione già a partire dalle scuole di specializzazione, che possa rafforzare le conoscenze del giovane nefrologo, incrementa le sue incertezze al momento del lavoro sul campo a contatto con i pazienti e incrementa le "controindicazioni relative" all'avvio del trattamento peritoneale, nonostante la possibilità di soddisfare le necessità cliniche e sociali dei pazienti.

Inoltre, si deve citare l'assenza di un'organizzazione ambulatoriale ben strutturata che accolga e guidi il paziente nel suo percorso di conoscenza della metodica.

L'orientamento alla scelta deve partire dall'educazione del paziente già in fase predialitica. A tale scopo, è utile un ambulatorio pre-dialisi ben organizzato, con del personale medico-infermieristico motivato e dedicato esclusivamente a tale compito. Solo in questo modo, l'ambulatorio diventa lo strumento indispensabile per presentare la metodica in maniera chiara e corretta, coinvolgendo non solo il paziente ma anche i familiari ed eventuali caregiver. Il tessuto sociale intorno al malato deve essere coinvolto in questo percorso, in quanto la dialisi domiciliare coinvolge in parte anche la famiglia. Tale entourage comprende anche il medico di medicina generale, il quale, conoscendo in prima persona e da più tempo il malato, diventa una figura di riferimento, la cui opinione conta e che 
l'equipe del centro dialisi non può ignorare.

Infine, è da notare la mancanza di personale infermieristico adeguatamente formato, con esperienza e dedicato esclusivamente alla dialisi peritoneale. Gli infermieri sono il biglietto da visita del centro dialisi e sono fondamentali nel legame tra malato e dialisi.

Certamente, la crescente fragilità dei pazienti avviati alla dialisi e la sempre maggiore difficoltà a reperire un supporto familiare nella gestione domiciliare fanno sì che, in questo percorso, sia necessaria la percezione della sempre presente disponibilità del centro e del supporto infermieristico e medico, in qualsiasi momento il paziente o il caregiver ne abbiano bisogno.

Ulteriori investimenti ad ampio spettro sono, però, necessari per risollevare le sorti della dialisi peritoneale:

maggiori investimenti nel campo della ricerca di base, per chiarire in modo capillare i meccanismi del trasporto peritoneale e rendere le soluzioni sempre più biocompatibili, riducendo, in questo modo, i danni (immunologici e funzionali) alla membrana peritoneale;

rafforzare la formazione degli operatori medici e infermieri. Interrompere il "circolo vizioso" che può venirsi a creare in quei centri che, per l'esiguo numero di pazienti, si trovano a non essere in grado di gestire in modo adeguato non solo l'addestramento e la cura del paziente, ma anche le possibili complicanze medico-infermieristiche, proprio per la scarsa casistica;

creare strategie alternative, come una "rete" tra nefrologi "peritonealisti", per scambiarsi dubbi e opinioni, che possa superare la problematica dei piccoli numeri, in modo da poter imparare a gestire nel migliore dei modi il trattamento. Continuare a occuparsi o riappropriarsi (come nefrologi) della tecnica di posizionamento del catetere peritoneale, spesso facilmente demandata al chirurgo;

incentivare i servizi sul territorio, sia da un punto di vista economico che gestionale, con contributi ai pazienti avviati alla dialisi domiciliare ma, soprattutto, con la creazione di strutture sanitarie adibite a "case della salute" in cui i pazienti senza supporto familiare possano trovare un sostegno, soprattutto infermieristico, per le problematiche della vita quotidiana.

\section{Riassunto}

La dialisi peritoneale offre diversi vantaggi rispetto all'emodialisi, inclusa una migliore qualità di vita. Nonostante la sua evoluzione scientifica e tecnologica, resta una metodica marginale. A nostro avviso, aIcuni dei principali fattori responsabili della bassa penetranza della dialisi peritoneale sono da ricercare nella debole motivazione dei nefrologi, appesantita da un aggiornamento spesso inadeguato di medici e infermieri, dalla scarsa formazione dei medici specializzandi e dalla mancanza di un ambulatorio correttamente organizzato con personale motivato e dedicato. Ulteriori investimenti ad ampio spettro sono, quindi, necessari per risollevare le sorti della dialisi peritoneale; tra essi, l'investimento sulla ricerca, sulla formazione e sull'integrazione tra ospedale e territorio.

Parole chiave: Assistenza integrata, Addestramento, Formazione, Territorio

Dichiarazione di conflitto di interessi: Gli Autori dichiarano di non avere conflitto di interessi.

Contributi economici degli autori: Gli Autori dichiarano di non aver ricevuto sponsorizzazioni economiche per la preparazione dell'articolo.

Indirizzo degli Autori:

Dr. Renzo Scanziani

Divisione di Nefrologia e Dialisi

Azienda Ospedaliera di Desio e Vimercate

Presidio di Desio

Via Mazzini 1

20832 Desio (MB)

renzo.scanziani@aovimercate.org 